\section{Autoantikörper gegen Myelin- Oligodendrozyten-Glykoprotein}

\author{
W. Stöcker \\ Euroimmun Medizinische Labordiagnostika AG, Lübeck, \\ Deutschland
}

Synonym(e) Antikörper gegen MOG; Anti-MOG-Antikörper

Englischer Begriff antibodies against myelin oligodendrocyte glycoprotein; anti-myelin oligodendrocyte glycoprotein antibodies; anti-MOG antibodies

Definition Autoantikörper gegen ein integrales Membranprotein des Zentralnervensystem-(ZNS-)Myelins. MOG wird ausschließlich in myelinisierenden Oligodendrozyten exprimiert. Es ist in der Oligodendrozyten-Plasmamembran und auf der extrazellulären Seite der äußersten Myelinlamelle lokalisiert, fehlt aber weitgehend im kompakten Myelin. Sein Anteil am gesamten Myelinprotein beträgt 0,01-0,05 \%.

Funktion - Pathophysiologie Bei der Entstehung entzündlicher demyelinisierender Erkrankungen des ZNS wird Autoantikörpern gegen MOG eine mögliche immunpathogene Rolle zugeschrieben bzw. MOG als ein relevantes Zielantigen autoreaktiver T- und B-Zellen angesehen. Das Potenzial von Anti-MOG-Antikörpern zur Induktion der Demyelinisierung wurde an Hirnzellkulturen und im Tiermodell für autoimmune Enzephalomyelitis demonstriert.

Analytik Die Bestimmung von Autoantikörpern gegen Myelin-Oligodendrozyten-Glykoprotein sollte mit Testsystemen erfolgen, die MOG mit authentischer, membranständiger Konformation und nativer Glykosylierung als Antigensubstrat verwenden. Geeignet ist der indirekte Immunfluoreszenztest ( Immunfluoreszenz, indirekte) mit MOG-transfizierten HEK293-Zellen.
Untersuchungsmaterial Serum, Plasma oder Liquor.

Probenstabilität Autoantikörper sind bei $+4{ }^{\circ} \mathrm{C}$ bis $\mathrm{zu}$ 2 Wochen lang beständig, bei $-20^{\circ} \mathrm{C}$ über Monate und Jahre hinweg.

Diagnostische Wertigkeit Autoantikörper gegen MyelinOligodendrozyten-Glykoprotein werden bei einem Teil der Patienten mit Entmarkungserkrankungen des ZNS gefunden, insbesondere in pädiatrischen Fällen. Dazu gehören die akute disseminierte Enzephalomyelitis (ADEM) und das ,klinisch isolierte (episodische, demyelinisierende) Syndrom“. Des Weiteren finden sich die Antikörper bei Anti-AQP4-negativen Patienten mit NMO-Spektrum-Erkrankungen (Optikusneuritis und/oder longitudinale extensive transverse Myelitis), sie sind hier indikativ für einen monophasischen Krankheitsverlauf und eine bessere Prognose.

\section{Literatur}

Jarius S, Ruprecht K, Kleiter I, Borisow N, Asgari N, Pitarokoili K, Pache F, Stich O, Beume LA, Hümmert MW, Trebst C, Ringelstein M, Aktas O, Winkelmann A, Buttmann M, Schwarz A, Zimmermann H, Brandt AU, Franciotta D, Capobianco M, Kuchling J, Haas J, Korporal-Kuhnke M, Lillevang ST, Fechner K, Schanda K, Paul F, Wildemann B, Reindl M (2016) MOG-IgG in NMO and related disorders: a multicenter study of 50 patients. Part 1: frequency, syndrome specificity, influence of disease activity, longterm course, association with AQP4-IgG, and origin. J Neuroinflammation 13(1):279

McLaughlin KA, Chitnis T, Newcombe J et al (2009) Age-dependent $B$ cell autoimmunity to a myelin surface antigen in pediatric multiple sclerosis. J Immunol 183:4067-4076

Pröbstel AK, Dornmair K, Bittner R, Sperl P, Jenne D, Magalhaes S, Villalobos A, Breithaupt C, Weissert R, Jacob U et al (2011) Antibodies to MOG are transient in childhood acute disseminated encephalomyelitis. Neurology 77:580-588 